\title{
Formulación del problema de optimización multiobjetivo del confort en edificación sostenible
}

\author{
M. Castilla ${ }^{a, *}$, F. Rodríguez ${ }^{b}$, J.D. Álvarez ${ }^{b}$, M. Berenguel $^{b}$, C. Bordons $^{a}$ \\ ${ }^{a}$ Universidad de Sevilla. Dpto. de Ingeniería de Sistemas y Automática, Sevilla (España) \\ ${ }^{b}$ Universidad de Almería - CIESOL - ceiA3. Dpto. de Informática, Almería (España)
}

\begin{abstract}
Resumen
En este trabajo se realiza un análisis detallado del problema de optimización del confort desde un enfoque basado en criterios de edificación sostenible. Para ello, se propone una arquitectura de control jerárquico multicapa gobernada mediante una estrategia de optimización multiobjetivo que proporciona trayectorias de referencia para la temperatura del aire y la iluminancia en el interior de las estancias de un edificio. Los objetivos que se han establecido para el desarrollo de este trabajo son maximizar el confort térmico, el confort visual y la eficiencia energética.
\end{abstract}

Palabras clave: Edificación sostenible; confort; eficiencia energética; optimización multiobjetivo

\section{Introducción}

La creciente preocupación por el cambio climático, principalmente derivado de las actividades desarrolladas por las personas, así como el elevado consumo energético y su coste asociado han dado lugar a la aparición de distintas estrategias, como por ejemplo la estrategia Europa H2020 [5], que intenta reducir las emisiones de $\mathrm{CO}_{2}$ y aumentar el ratio de fuentes de energía renovables. Por otro lado, según [6], los edificios (residenciales y del sector servicios) representan el $36 \%$ del consumo energético total de la Unión Europea, lo que ha ocasionado la aparición de diversas normativas relativas a la eficiencia energética de los edificios (nuevos o rehabilitados) en base a las condiciones climáticas exteriores, así como, a los requisitos interiores en términos de coste-efectividad.

De este modo, surge el concepto de edificación sostenible cuyo objetivo principal es concebir los edificios de manera sustentable, es decir, optimizando el uso de recursos naturales y los elementos estructurales del propio edificio para minimizar el impacto de los mismos sobre los usuarios y el entorno que lo rodea. Por lo tanto, este nuevo paradigma solo sería alcanzable a través de una gestión integral y óptima de todos los insumos propios de los procesos constructivos y, especialmente, de los insumos asociados a su uso a lo largo de su ciclo de vida, entre los que la energía para el acondicionamiento ambiental resulta crucial tanto por su orden de magnitud como por su repercusión en el bienestar de los ocupantes.

En general, a la hora de mejorar el comportamiento de un edificio desde el punto de vista de la eficiencia energética es necesario considerar tres factores distintos: los elementos estructurales del edificio, su uso y los usuarios del mismo. De esta manera, hay que buscar una solución de compromiso entre eficiencia energética y el confort de los usuarios. Para ello, el clima interior del edificio debe regularse intentando aprovechar las ventajas proporcionadas por la propia arquitectura del edificio y el clima característico del lugar donde éste se encuentre localizado. Sin embargo, la eficiencia energética y la regulación del clima interior son criterios opuestos debido, principalmente, a que para conseguir una situación de confort óptima para los usuarios de un edificio puede ser necesario incrementar el consumo de energía. Por lo tanto, el control eficiente del confort en edificios es un problema de optimización multiobjetivo.

Son muchos los autores que han analizado la optimización de la energía y/o el confort en las distintas fases de un edificio, comprendiendo desde su diseño hasta su uso a lo largo de su ciclo de vida. Por ello, es posible encontrar diversos trabajos en la literatura que optimizan uno o varios criterios en la fase de diseño, como por ejemplo confort térmico y eficiencia energética [15]. También es posible encontrar trabajos de optimización multiobjetivo que permiten evaluar distintas modificaciones arquitectónicas en el proceso de rehabilitación de edificios y su influencia en el confort de los usuarios y el consumo de energía [2]. Asimismo, existen otros trabajos que optimizan el funcionamiento de sistemas representativos del edificio, como por ejemplo el sistema de climatización [4].

Como se puede observar, la mayor parte de los trabajos están enfocados a mejorar algún aspecto concreto en el diseño y construcción de edificios. Sin embargo, también es necesario optimizar el comportamiento de edificios ya construidos en los cuales no es posible modificar su arquitec- 
tura o incorporar nuevos elementos estructurales que permitan disminuir la diferencia existente entre maximización del confort y minimización del consumo de energía. En este trabajo se presenta la formulación multiobjetivo para la optimización del confort de los usuarios en edificación sostenible. Para ello, se proponen tres objetivos claramente diferenciados: confort térmico, confort visual y eficiencia energética. Además, también se muestra detalladamente su integración en una arquitectura jerárquica multicapa. Más concretamente, la formulación del problema multiobjetivo presentada en este trabajo se ha personalizado para el edificio CIESOL (www.ciesol.es), véase figura 1.

La estructura de este artículo es la siguiente: la sección 2 incluye una explicación detallada de la formulación del problema de optimización multiobjetivo incluyendo la relación existente entre las funciones objetivo y las variables de decisión seleccionadas. Por otro lado, la sección 3 describe la arquitectura jerárquica multicapa propuesta para la resolución del problema de optimización multiobjetivo formulado en este trabajo. Finalmente, en la sección 4, se incluyen las principales conclusiones y trabajos futuros.

\section{Optimización multiobjetivo en edificación sostenible: confort y eficiencia energética}

Un problema de optimización multiobjetivo se puede definir como la búsqueda de un vector de variables de decisión capaz de satisfacer ciertas restricciones y, al mismo tiempo, optimizar un vector cuyos elementos representan funciones objetivo [13]. Por lo tanto, se puede establecer que un problema de optimización multiobjetivo se caracteriza por tener dos o más objetivos contrapuestos (en conflicto) que deben ser minimizados (o maximizados) simultáneamente satisfaciendo ciertas restricciones [9], formulados en (1).

$$
J=\min _{x \in X}\left(J_{1}(x), J_{2}(x), \ldots, J_{n}(x)\right)
$$

sujeto a

$$
\begin{aligned}
& g_{i}(x) \in 0, \quad i=1,2, \ldots, m \\
& h_{i}(x)=0, \quad i=1,2, \ldots, p
\end{aligned}
$$

En este tipo de problemas no es posible encontrar una solución ideal capaz de optimizar todas las funciones objetivo. Sin embargo, si es posible hallar un conjunto de soluciones no dominantes conocido como "Frente óptimo de Pareto" [9], a partir del cual, se puede seleccionar una solución de compromiso. Concretamente, dicha elección se realiza mediante el uso técnicas de decisión basadas en diferentes criterios, como por ejemplo, esta- bleciendo restricciones en torno a los valores límite de temperatura o de iluminancia permitidos.

Una parte de vital importancia en problemas de optimización multiobjetivo es la definición del problema, es decir, es necesario especificar de forma adecuada las funciones objetivo, determinando las variables que las componen y estableciendo una relación de las mismas con las variables de decisión, que es lo que se pretende con el desarrollo de este trabajo. Para ello, se debe tener un conocimiento detallado de los procesos involucrados que permita precisar de manera clara y concisa las relaciones existentes entre los distintos objetivos.

Desde el punto de vista de la edificación sostenible, uno de los factores más analizados es la optimización del consumo energético. Sin embargo, también hay que considerar el confort de los usuarios como un factor a optimizar ya que el bienestar de una persona está directamente relacionada con su productividad [7]. Por lo tanto, en este trabajo se ha considerado que el problema de optimización multiobjetivo está compuesto por tres objetivos que deben ser maximizados: confort térmico, confort visual y eficiencia energética. Asimismo, la solución de este problema de optimización multiobjetivo, $x \forall \mathbf{X}$, proporcionará las trayectorias de referencias presentes y futuras de temperatura interior, $\mathbf{X}_{T A}$, e iluminancia, $\mathbf{X}_{I L}$, véase (2).

$$
x=\left[\mathbf{X}_{T A}, \mathbf{X}_{I L}\right] \forall \mathbf{X}
$$

En la ecuación anterior $\mathbf{X}_{T A}$ y $\mathbf{X}_{I L}$ representan vectores de referencias presentes y futuras de temperatura interior e iluminancia a lo largo del horizonte de optimización.

Además, para resolver el problema de optimización multiobjetivo propuesto en este trabajo es necesario disponer de modelos para: i) estimar el comportamiento climático en el interior de un edificio y el confort de los usuarios y ii) establecer las relaciones entre funciones objetivo y variables de decisión. Más concretamente, el comportamiento dinámico del clima en el interior del edificio se ha caracterizado mediante un modelo basado en primeros principios [3]. Por otra parte, el confort de los usuarios (térmico y visual) se ha modelado utilizando diversos índices recomendados por estándares internacionales: voto medio estimado (PMV, siglas del inglés: Predicted Mean Vote) para el confort térmico [8] y diferencia de iluminancia $\left(D_{i}\right)$ para el confort visual [3]. Dichos índices permiten establecer una relación directa entre el confort de los usuarios y las principales variables climáticas en el interior de un edificio. De este modo, el confort de los usuarios en función del clima interior se puede describir mediante dos modelos dinámicos representados por dos sistemas de ecuaciones 

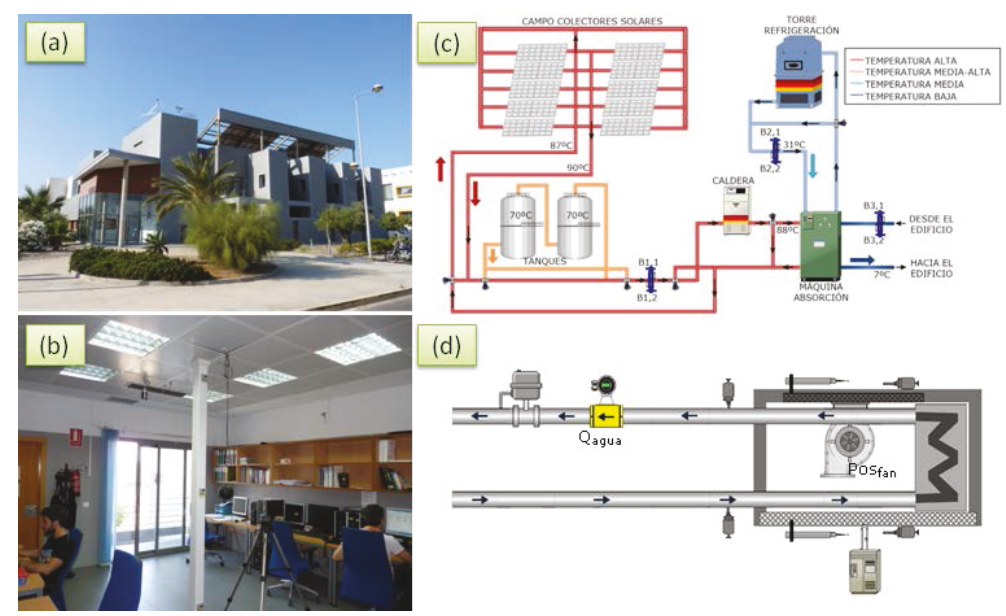

(d)

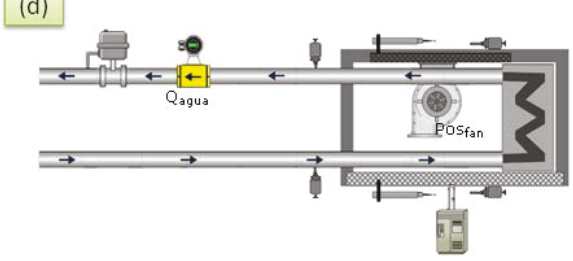

Figura 1: Planta de ensayo: CIESOL. (a) Exterior del edificio, (b) Habitación característica, (c) Esquema de la instalación de frío solar, (d) Esquema de un fancoil

diferenciales, véase (3)-(4).

$$
\begin{aligned}
\frac{d \mathbf{X}_{c l}}{d t} & =f_{c l}\left(\mathbf{X}_{c l}, \mathbf{X}_{c n f}, \mathbf{U}, \mathbf{D}, \mathbf{V}, \mathbf{C}, t\right) \\
\frac{d \mathbf{X}_{c n f}}{d t} & =f_{c n f}\left(\mathbf{X}_{c l}, \mathbf{X}_{c n f}, \mathbf{U}, \mathbf{D}, \mathbf{V}, \mathbf{C}, t\right)(4)
\end{aligned}
$$

En las ecuaciones anteriores, $\mathbf{X}_{c l}=\mathbf{X}_{c l}(t)$ es un vector que contiene las principales variables de estado relacionadas con el clima de la habitación (temperatura, humedad relativa e iluminancia); $\mathbf{X}_{c n f}=\mathbf{X}_{c n f}(t)$ es un vector de las variables de estado de confort (PMV y $D_{i}$ ); $\mathbf{U}=\mathbf{U}(t)$ es un vector que representa las variables de entrada, en este trabajo, el sistema de climatización de frío solar (controlado por medio de la velocidad del fancoil $\left(\right.$ Pos $\left._{f a n}\right)$ y el caudal de agua que circula a través de él $\left(Q_{\text {agua }}\right)$ ), la ventana (controlada por su grado de apertura $\left.\left(A p_{\text {vent }}\right)\right)$, la persiana (controlada, al igual que en el caso de la ventana, por su grado de apertura $\left(A p_{\text {pers }}\right)$ y las luminarias (controladas por el grado de intensidad $\left(G r\right.$ Int $\left._{\text {lum }}\right)$ al que se encuentran reguladas); $\mathbf{D}=\mathbf{D}(t)$ es un vector que contiene las perturbaciones a las que está sometido el problema (temperatura exterior, humedad exterior, radiación, velocidad y dirección del viento exterior, número de personas en la habitación, etc.); $\mathbf{V}=\mathbf{V}(t)$ y $\mathbf{C}$ son vectores que contienen las variables y constantes del sistema, respectivamente; $t$ es el tiempo. Finalmente, $f_{c l}$ y $f_{c n f}$ son funciones no lineales basadas en balances de masa y transferencia de calor.

Una explicación más detallada de dichos modelos, los procesos involucrados y los parámetros asociados con los mismos se puede encontrar en [3]. En las siguientes secciones, se incluye una descripción detallada de los distintos objetivos a optimizar y de cómo pueden formularse en función de las variables de decisión seleccionadas en el problema de optimización (referencias presentes y futuras de temperatura interior e iluminancia).

\subsection{Maximización del confort térmico}

El término confort térmico se define como "Aquella condición de la mente que expresa satisfacción con el ambiente térmico" [8]. De acuerdo con esta definición, el confort térmico es un proceso cognitivo que depende de factores físicos, fisiológicos e incluso psicológicos. Sin embargo, es posible encontrar en la literatura diversos índices para estimar la sensación de confort térmico de grupos de personas sometidas a ciertas condiciones termohigrométricas. En este trabajo, se ha seleccionado el índice PMV [8], véase (5).

$$
P M V(t)=0,303 \exp ^{-0,036 M}+0,028 \sum L
$$

donde $M$ representa la actividad realizada por las personas en $\left(W / m^{2}\right)$ y $L$ es el balance térmico existente entre el cuerpo humano y en entorno que lo rodea cuando la persona está en una situación de confort $\left(W / m^{2}\right)$. Más concretamente, este balance térmico, $L$, está relacionado con la temperatura interior de la habitación mediante una función no lineal $L=f_{P M V}\left(\mathbf{X}_{T A}\right)$ [3].

El índice PMV proporciona un valor numérico de acuerdo a una escala de sensación térmica compuesta por siete estados: 0 situación confortable, $\leq 1$ ligeramente caluroso/fresco, $\leq 2$ calor/frío y $\leq 3$ mucho calor/frío. En general, los distintos estándares internacionales como el ISO 7730 [8] recomiendan mantener para una situación de confort clase B el índice PMV a 0 con una tolerancia de $\leq 0.5$. Por lo tanto, para maximizar el confort térmico hay que minimizar la función objetivo $J_{1}$, véase (6).

$$
J_{1}={ }_{t_{i}}^{t_{f}} \operatorname{PMV}(t) d t
$$




$$
\begin{aligned}
\text { sujeto a } & \\
P M V_{\min } & \geq P M V_{j}(t) \geq P M V_{\max } \\
\mid j & =t_{i}, \ldots, t_{f}
\end{aligned}
$$

donde $t_{i}$ y $t_{f}$ representan el intervalo de optimización. Por otra parte, $P M V_{\min }$ y $P M V_{\max }$ están determinados por la clase de confort térmico que se desea alcanzar. Por ejemplo, para una situación de confort clase B, $P M V_{\min }$ sería igual a 0,5 y $P M V_{\max }$ a 0,5 .

\subsection{Maximización del confort visual}

Según el estándar internacional UNE EN-12665 [1] el confort visual se puede definir como una "condición subjetiva de bienestar originada por el entorno visual". Por lo tanto, a partir de esta definición se puede deducir que es posible evaluar el confort visual a partir de algunas de las propiedades físicas del entorno, como por ejemplo el nivel de iluminancia, su distribución, el brillo, el color de la luz, la cantidad de luz natural en el ambiente, etc. En este trabajo, se ha definido una situación de confort visual en función del nivel de iluminancia existente en la habitación. Para ello, se va a utilizar el índice $D_{i}$ propuesto en [3], véase (7). Este índice representa la diferencia entre el nivel de iluminancia óptimo $\left(X_{I L_{o p t}}\right)$ y el existente en la habitación $\left(\mathbf{X}_{I L}\right)$ en función de la actividad que se esté realizando en el interior de la misma. Por ejemplo, para actividades típicas de oficina los diferentes estándares recomiendan mantener un nivel de iluminancia aproximado de 500 lux [1].

$$
D_{i}(t)=X_{I L_{o p t}} \quad X_{I L}(t)
$$

Por lo tanto, para maximizar el confort visual, la función objetivo $J_{2}$, que se puede observar en (8), debe ser minimizada. Al igual que en el caso del confort térmico, se ha considerado que el índice $D_{i}$ debe mantenerse dentro de un límite definido por $\left[0, D_{i_{\max }}\right]$.

$$
\begin{aligned}
& \text { sujeto a } \\
& \begin{aligned}
D_{i_{j}}(t) & \geq D_{i_{\max }} \\
\mid j & =t_{i}, \ldots, t_{f}
\end{aligned}
\end{aligned}
$$$$
J_{2}={ }_{t_{i}}^{t_{f}} D_{i}(t) d t
$$

donde $D_{i_{\max }}$ representa la diferencia máxima permitida entre el nivel de iluminación óptimo y el existente en la habitación.

\subsection{Minimización del coste del consumo de energía}

Para definir correctamente la función objetivo relacionada con la eficiencia energética, se deben tener en cuenta las características del recinto para el cual se está planteando el problema de optimización multiobjetivo. Como se mencionó anteriormente, en este caso el problema de optimización multiobjetivo se ha particularizado para un recinto característico del edificio CIESOL, véase figura 1. Por lo tanto, la función objetivo para la minimización del consumo de energía debe considerar el consumo de los diferentes actuadores disponibles, es decir, la ventana, la persiana, las luminarias y el sistema de climatización basado en frío solar. Asimismo, este edificio cuenta con un campo fotovoltaico para la generación de energía eléctrica, lo que supondrá una reducción en el coste de la energía eléctrica consumida de la red.

Por otro lado, también es necesario tener presente que el precio de la energía no es constante a lo largo del día, sino que varía en función de la oferta y la demanda. En general, los precios de la energía en Europa se fijan diariamente a las 12:00 horas para las 24 horas del día siguiente (Mercado diario) [10]. Posteriormente, ese precio se puede ajustar por medio de un mercado intradiario para garantizar el acoplamiento entre la oferta y la demanda. De esta manera, la función objetivo que representa el coste del consumo de energía para un horizonte de optimización $\left[t_{i}, t_{f}\right]$ se puede expresar tal y como se puede observar en (9):

$$
\begin{aligned}
& J_{3}={ }_{t_{i}}^{t_{f}} C_{e e}(t) E_{\text {pers }}(t) d t \\
& +{ }_{t_{i}}^{t_{f}} C_{e e}(t) E_{\text {vent }}(t) d t \\
& t_{f} \\
& +{ }_{t_{i}} C_{e e}(t) E_{l u z}(t) d t \\
& +{ }_{t_{i}}^{t_{f}} C_{e e}(t) E_{f a n}(t) d t \\
& t_{i} \\
& t_{f} \\
& +{ }_{t_{i}}^{t_{f}} C_{e e}(t) Q_{\text {agua }}(t) E_{\text {frio }}(t) d t
\end{aligned}
$$

En la ecuación anterior $E_{\text {pers }}, E_{v e n t}, E_{\text {luz }}$ y $E_{\text {fan }}$ representan el consumo en $(k W h)$ asociado con la persiana, la ventana, las luminarias y el ventilador del sistema de climatización respectivamente. $E_{\text {frio }}$ es el consumo necesario para calentar/enfriar 1 litro de agua utilizando la instalación de frío solar en $(k W h / l)$ y $Q_{a g u a}$ es el consumo de agua por parte del fancoil en $l / \min$. Finalmente, $C_{e e}$ es el coste de la energía en $(€ / k W h)$. Sin embargo, para calcular el coste total de la energía eléctrica $\left(C_{e e}\right)$ hay que tener en cuenta que ratio de dicha energía ha sido proporcionada por la instalación fotovoltaica y cual por la red, véase (10).

$$
\begin{aligned}
C_{e e}(t) & =\left(\begin{array}{lll}
1 & \alpha
\end{array}\right){ }_{t_{i}}^{t_{f}} C_{e e, r e d}(t) d t \\
& +\quad \alpha{ }_{t_{i}}^{t_{f}} C_{e e, f o t}(t) d t
\end{aligned}
$$


donde $\alpha$ es el ratio de energía procedente de la instalación fotovoltaica, $C_{e e, r e d}$ es el coste de la energía eléctrica suministrada por la red (este coste depende de los precios horarios fijados en los mercados diario e intradiarios) y $C_{e e, f o t}$ es el coste asociado con la utilización de la energía proporcionada por la instalación fotovoltaica.

A continuación, se muestra un estudio detallado de cada uno de los términos que aparecen en la ecuación (9). Más concretamente, se analizan las variables que intervienen en los mismos y su relación con las variables de decisión (temperatura del aire e iluminancia).

Consumo de energía de la persiana: Está directamente relacionado con el motor que se encarga de la apertura/cierre automático de la misma. Específicamente, dicho consumo depende de la potencia del motor $\left(P_{\text {mot,pers }}\right)$ y del tiempo que se esté usando el mismo ( $\left.t_{\text {uso, mot,pers }}\right)$, tal y como se muestra en (11). Asimismo, $t_{u s o, m o t, p e r s}$ se puede calcular en función de los cambios en el grado de apertura de la persiana. La relación existente entre la temperatura del aire $\left(\mathbf{X}_{T A}\right)$ y la iluminancia $\left(\mathbf{X}_{I L}\right)$ con el grado de apertura de la persiana $\left(A p_{\text {pers }}\right)$ se ha determinado a través de un modelo basados en primeros principios $\left(f_{1}\right)$, véase figura 2. Más concretamente, en ambos casos el grado de apertura de persiana es la variable de control que se utiliza para amortiguar la influencia de la radiación exterior (luz natural) sobre las variables de decisión.

$$
\begin{aligned}
E_{\text {pers }}(t) & =P_{\text {mot,pers }} t_{\text {uso }, \text { mot,pers }} \\
t_{\text {uso }, \text { mot }, \text { pers }} & =0,155 \frac{d A p_{\text {pers }}(t)}{d t}
\end{aligned}
$$

donde,

$$
\frac{d A p_{\text {pers }}(t)}{d t}=f_{1}\left(\mathbf{X}_{T A}, \mathbf{X}_{I L}, t\right)
$$

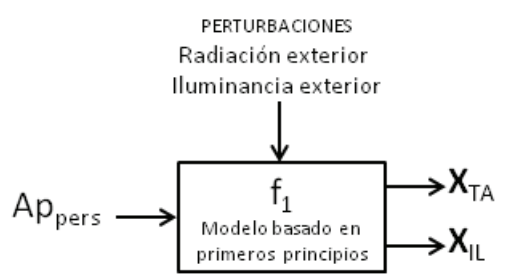

Figura 2: Relación de la variable de actuación $A p_{\text {pers }}$ y las variables de decisión $\mathbf{X}_{T A}$ y $\mathbf{X}_{I L}$

Consumo de energía de la ventana: Al igual que en el caso anterior, el consumo relacionado con la ventana está directamente asociado con el motor que se encarga de la apertura/cierre automática de la misma. Por lo tanto, dicho consumo depende de la potencia del motor $\left(P_{\text {mot,vent }}\right)$ y del tiempo que se esté usando el mismo $\left(t_{\text {uso,mot,vent }}\right)$, véase (12). Asimismo, $t_{u s o, m o t, v e n t}$ se puede calcular en función de los cambios en el grado de apertura de la ventana $\left(A p_{\text {vent }}\right)$ mediante una función no lineal $\left(f_{2}\right)$. Más concretamente, dicha función representa un modelo basado en primeros principios del proceso de ventilación natural que se produce a través de la ventana el cuál, a su vez, depende de la temperatura del aire $\left(\mathbf{X}_{T A}\right)$ y está influenciado por la temperatura exterior y la velocidad y dirección del viento, véase figura 3.

$$
\begin{aligned}
E_{\text {vent }}(t) & =P_{\text {mot,vent }} t_{\text {uso }, \text { mot }, \text { vent }} \\
t_{\text {uso }, \text { mot }, \text { vent }} & =0,155 \frac{d A p_{\text {vent }}(t)}{d t}
\end{aligned}
$$

donde,

$$
\frac{d A p_{\text {vent }}(t)}{d t}=f_{2}\left(\mathbf{X}_{T A}, t\right)
$$

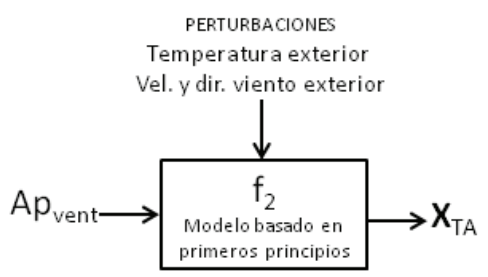

Figura 3: Relación de la variable de actuación $A p_{\text {vent }}$ y la variable de decisión $\mathbf{X}_{T A}$

Consumo de energía de las luminarias: Para estimar el consumo de energía por parte de las luminarias hay que tener en cuenta que, en el caso del edificio CIESOL, es posible modificar su intensidad luminosa mediante reguladores que han sido instalados. De esta forma, los factores que intervienen en el consumo de energía de las luminarias $\left(E_{\text {luz }}\right)$ son el número de luminarias encendidas, la potencia de las mismas y la intensidad a la que cada una de ellas se encuentra regulada, véase (13). Por otro lado, el número de luminarias encendidas $\left(N_{l}\right)$ y la intensidad a la que se regulan $\left(G r I n t_{\text {lum }}\right)$ tienen una influencia directa sobre la iluminancia interior $\left(\mathbf{X}_{I L}\right)$. Dicha influencia se ha modelado mediante un modelo de caja negra $\left(f_{3}\right)$, véase figura 4.

$$
E_{\text {luz }}(t)=\int_{j=1}^{N_{l}} P_{\text {lum }_{i}} \operatorname{GrInt}_{\text {lum }_{i}}(t)
$$

donde $N_{l}$ representa el número de luminarias que se encuentran encendidas en la habitación ( ), $P_{\text {lum }}$ es la potencia asociada con cada una de las luminarias en $(W)$ y $G r$ Int $_{\text {lum }_{i}}$ es el grado de intensidad al que se encuentra regulada cada una de las luminarias en (\%). 


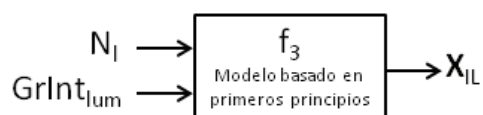

Figura 4: Relación de las variables de actuación $N_{l}$ y $G r I_{n} t_{l u m_{i}}$ y la variable de decisión $\mathbf{X}_{I L}$

Consumo de energía del sistema de climatización: El edificio para el cual se está planteando el problema de optimización multiobjetivo cuenta con un sistema de climatización basado en frío solar. Más concretamente, dicha instalación está compuesta por un campo de colectores solares, un sistema de acumulación de agua caliente, una caldera y una máquina de absorción con su torre de refrigeración, véase figura 1 (c) que se encargan de calentar/enfriar agua en base al modo de operación de la planta (invierno/verano). Posteriormente, ese agua circula por el edificio a través de los fancoils instalados en cada una de las habitaciones. En la figura 1(d) se muestra el esquema general de un fancoil. Por lo tanto, para regular el aporte de frío/calor a la habitación por parte del fancoil instalado se disponen de dos grados de libertad: la velocidad del ventilador $\left(\operatorname{Pos}_{f a n}\right)$ y el caudal de agua que circula a través del fancoil $\left(Q_{\text {agua }}\right)$. De esta manera, para estimar el consumo de energía total del sistema de climatización, se tendrán que considerar dos elementos: el consumo del ventilador, véase (14), y el consumo asociado con la propia instalación de frío solar para enfriar/calentar el agua que circula a través de los fancoils, (15). Además, es necesario mencionar que dicho aporte está directamente relacionado con la temperatura del aire, $\mathbf{X}_{T A}$, véase figura 5 . En particular, la instalación de frío solar es la responsable de proporcionar la cantidad deseada de agua a una cierta temperatura $\left(T_{\text {agua }}\right)$ al fancoil instalado en la habitación. Posteriormente, dicho fancoil es capaz de influir mediante un proceso de ventilación forzada en la variable de decisión $\mathbf{X}_{T A}$ introduciendo aire en la habitación a una determinada temperatura $\left(T_{i m p}\right)$. Finalmente, esa temperatura se puede controlar a través de las variables de actuación $Q_{a g u a}$ y $\operatorname{Pos}_{f a n}$.

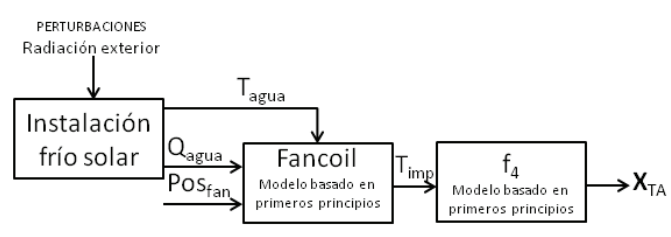

Figura 5: Relación de las variables de actuación $Q_{a g u a}$ y $P_{\text {os }}$ yn y la variable de decisión $\mathbf{X}_{T A}$

En el primer caso, el consumo del ventilador $\left(E_{f a n}\right)$ depende de la posición del mismo $\left(\right.$ Pos $\left._{f a n}=0 \% \quad 33 \% \quad 66 \% \quad 100 \%\right)$, de la potencia absorbida por el ventilador para cada una de las posiciones mencionadas anteriormente $\left(P_{a b s, f a n}\right)$ y del tiempo de uso $\left(t_{u s o, f a n}\right)$.

$$
E_{f a n}(t)=\operatorname{Pos}_{f a n} P_{a b s, f a n} t_{u s o, f a n}
$$

Por otro lado, para calcular el consumo asociado con la instalación de frío solar, se han tenido en cuenta los elementos con un consumo de energía mayor, véase figura 1(c), es decir, las principales bombas, la caldera, la torre de refrigeración y la máquina de absorción. De esta forma, dicho consumo puede expresarse en función del estado de esos elementos $(\mathrm{On} / \mathrm{Off})$ y del consumo característico de los mismos tal y cómo se puede observar en (15).

$$
\begin{aligned}
E_{\text {frio }}(t) & =E t_{B 1,1} P_{B 1,1}+E t_{B 1,2} P_{B 1,2} \\
& +E_{B 2,1} P_{B 2,1}+E s t_{B 2,2} P_{B 2,2} \\
& +E_{B 3,1} P_{B 3,1}+E s t_{B 3,2} P_{B 3,2} \\
& +E_{B 7} P_{B 7}+E s t_{\text {caldera }} P_{\text {caldera }} \\
& + \text { Est }_{\text {abs }} P_{\text {abs }} \\
& + \text { Est }_{\text {Torre }} P_{\text {Torre }}
\end{aligned}
$$

donde, las variables $E s t_{x}$ representan el estado $(1 / 0)$ del actuador $x$ asociado con los distintos actuadores que se pueden apreciar en la figura 1(c). Por otra parte, $P_{x}$ es la potencia de los mismos.

\subsection{Problema de optimización multiobjetivo}

El problema multiobjetivo definido en este trabajo está compuesto por las funciones objetivo definidas por las ecuaciones (6), (8) y (9). Como se ha mostrado en secciones anteriores, dichas funciones objetivo pueden expresarse, entre otras variables $(\Psi)$, en función de la temperatura interior de la habitación, $\mathbf{X}_{T A}$ y/o de la iluminancia $\mathbf{X}_{I L}$, es decir, las funciones objetivo se pueden expresar según (16):

$$
J_{i}\left(\mathbf{X}_{T A}, \mathbf{X}_{I L}, \Psi\right)=J_{i}(\mathbf{X}, \Psi), \quad \mid i=1,2,3
$$

Por otro lado, si se analizan los objetivos planteados en este trabajo, se puede observar que lo que se pretende es maximizar dos de ellos (confort térmico y confort visual) y minimizar el tercero (el coste del consumo de energía). Sin embargo, para satisfacer dichos objetivos es necesario minimizar las funciones objetivo asociadas con cada uno de ellos, véase (6), (8) y (9).

$$
J=\min _{x \in X}\left(J_{1}(\mathbf{X}, \Psi), J_{2}(\mathbf{X}, \Psi), J_{3}(\mathbf{X}, \Psi)\right)
$$




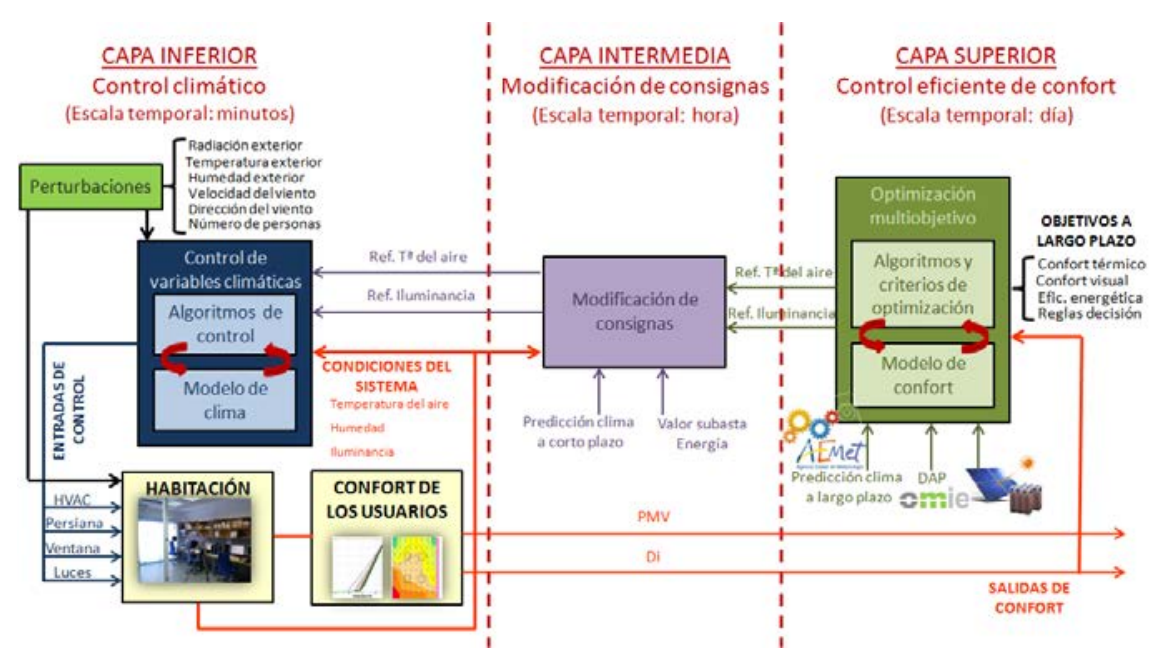

Figura 6: Arquitectura de control jerárquico multicapa

\section{Arquitectura de control jerárquico multicapa}

Las dinámicas involucradas en el proceso del control del confort en edificación sostenible presentan distintas escalas de tiempo tal y como se describió anteriormente. Por lo tanto, en este trabajo se propone una arquitectura de control jerárquico multicapa [14] donde las consignas para las distintas capas se obtienen de la resolución del problema de optimización multiobjetivo.

Más concretamente, la arquitectura que se propone en este trabajo está formada tres capas, véase figura 6. La capa superior (capa de la derecha en la figura 6), es la encargada de resolver el problema de optimización multiobjetivo presentado en la sección 2 en base a los objetivos definidos por (6), (8) y (9). Las salidas que proporciona esta capa son las consignas presentes y futuras de temperatura interior del aire, $\mathbf{X}_{T A}$, e iluminación, $\mathbf{X}_{I L}$, para la habitación. Sin embargo, esas trayectorias de referencia pueden modificarse en la capa intermedia (capa central en la figura 6) en función de las previsiones climáticas a corto plazo [12] y del precio real de venta de energía [10]. Finalmente, la capa inferior (capa más a la izquierda en la figura 6) contiene los algoritmos de control necesarios para minimizar el error entre las variables medidas en el interior de la habitación y las consignas estimadas por las capas superiores.

Capa superior: Control eficiente del confort. A partir de los objetivos marcados (confort térmico, confort visual y eficiencia energética) y las previsiones a largo plazo del precio de la energía y de las principales variables climáticas, se realiza el proceso de optimización para calcular las trayectorias de referencia de la temperatura interior y la iluminancia a lo largo del horizonte de control considerado (1 día) con un periodo de muestreo de 1 hora (48 variables de decisión).

Concretamente, las previsiones a largo plazo del precio de la energía se obtiene de [10]. Además, las previsiones de las principales variables climáticas, a lo largo del horizonte de control considerado por esta capa, se calculan utilizando una herramienta software que permite acceder a las previsiones proporcionadas por la Agencia Estatal de Meteorología (AEMET) para el día siguiente en función de varios índices (claridad, temperaturas mínima, media y máxima, y la radiación solar) [11]. El principal problema derivado del uso de predicciones a largo plazo es la incertidumbre que se introduce en el algoritmo de optimización. Sin embargo, esta incertidumbre se puede reducir gracias a la acción adaptativa proporcionada por la segunda capa.

Capa intermedia: Modificación de consignas. En esta capa, cada hora se modifican, si es necesario, las consignas estimadas por la capa superior al inicio del día en función de la predicción a corto plazo de las principales variables climáticas y del precio de la energía previsto según las modificaciones ocasionadas por las distintas sesiones del mercado intradiario [10]. Posteriormente, las nuevas consignas se envían a la capa inferior.

Capa inferior: Control climático. Finalmente, usando las referencias de temperatura interior del aire e iluminancia estimadas por las capas superiores, se calculan las señales de control adecuadas para los actuadores. Para ello, es posible utilizar una amplia gama de algoritmos de control, como por ejemplo, control adaptativo, control predictivo, control basado en reglas, etc. Sin embargo, el desarrollo de los algoritmos de control que se pueden utilizar para seguimiento de consignas en el ámbito del control eficiente del confort en edificios está fuera del ámbito de este trabajo. Una extensa 
revisión bibliográfica sobre este tipo de algoritmos de control se puede encontrar en [3].

\section{Conclusiones y futuros trabajos}

Actualmente, y debido fundamentalmente al cambio climático, la construcción y adaptación de edificios existentes a edificios denominados de consumo-cero están recibiendo gran atención por parte de empresas, investigadores e instituciones gubernamentales. En este trabajo, se han analizado los principales factores que incluyen en el confort de los usuarios desde un punto de vista de la edificación sostenible y se plantea una posible formulación del problema de optimización multiobjetivo considerando tres objetivos diferentes: confort térmico, confort visual y eficiencia energética. Asimismo, se ha propuesto una arquitectura de control jerárquica multicapa que permita la correcta resolución del problema de optimización multiobjetivo formulado en este trabajo.

Como trabajos futuros, se va a proceder a la implementación del problema de optimización multiobjetivo, y a su integración en la arquitectura de control propuesta. Actualmente, se ha finalizado la instalación de nuevos sensores y actuadores. Asimismo, se van a realizar ensayos reales en un recinto característico del edificio CIESOL.

\section{Agradecimientos}

M. Castilla quiere agradecer al Ministerio de Economía y Competitividad (Secretaría de Estado de Investigación, Desarrollo e Innovación) por financiar este trabajo a través del programa "Juan de la Cierva - Formación". J.D. Álvarez pertenece al programa del MEC "Ramón y Cajal" cofinanciado por la fundación social Europea. Ha sido posible realizar esta investigación gracias a la colaboración de la Fundación Iberdrola España a través de la Convocatoria de Ayudas a la Investigación en Energía y Medio Ambiente "Energía para la Investigación". Este trabajo ha sido parcialmente financiado con el Proyecto $\mathrm{R}+\mathrm{D}+\mathrm{i}$ del Plan Nacional DPI2014-56364-C2-1-R del Ministerio de Economía y Competitividad y Fondos FEDER.

\section{Referencias}

[1] EN 12665. Light and lighting - Basic terms and criteria for specifying lighting requirements. Brussels: European Commitee for Standardization, 2011.

[2] E. Asadi, M. Gameiro, Hengeller C. y L. Dias. A multi-objective optimization model for building retrofit strategies using TRNSYS simulations, GenOpt and matlab. Building and Environment, 56:370-378, 2012.
[3] M. Castilla, J.D. Álvarez, F. Rodríguez y M. Berenguel. Comfort control in buildings. Springer. Advances series in Industrial Control, 2014. ISBN 978-1-4471-6346-6.

[4] K. Dovrtel y S. Medved. Multi-objective optimization of a building free cooling system based on weather prediction. Energy and Buildings, 52:99-106, 2012.

[5] EUROPE 2020. ec.europa.eu/ europe2020/index_en.htm, 2016. Último acceso: 31 Mayo 2016.

[6] Eurostat. http://ec.europa.eu/eurostat, 2016. Último acceso: 31 Mayo 2016.

[7] A. Feige, H. Wallbaum, M. Janser y L. Windlinger. Impact of sustainable office buildings on occupant's comfort and productivity. Journal of Corporate Real State, 15 (1):7-34, 2013.

[8] ISO7730. Moderate thermal environments. Determination of the PMV and PPD indices and specification of the conditions for thermal comfort. International Organisation for Standardisation, 1994.

[9] G.P. Liu, J.B. Yang y J.F. Whidborne. Multiobjective optimisation and control. Recent Studies Press, 2003.

[10] Omie. http://www.omie.es, 2016. Último acceso: 06 Junio 2016.

[11] C. Osorio. Implantación y puesta a punto de un sistema de control jerárquico del crecimiento del cultivo de un invernadero regulando las variables climáticas. Proyecto Fin de Carrera, Universidad de Almería, España, 2011.

[12] A. Pawlowski, J. L. Guzmán, F. Rodríguez, M. Berenguel y J. Sánchez. Application of time-series methods to disturbance estimation in predictive control problems. En IEEE International Symposium on Industrial Electronics (ISIE), Bari, Italy. 2010.

[13] A. Ramírez-Arias, F. Rodríguez, J.L. Guzmán y M. Berenguel. Multiobjective hierarchical control architecture for greenhouse crop growth. Automatica, 48:490-498, 2012.

[14] F. Rodríguez. Modelado y control jerárquico de crecimiento de cultivos en invernadero. Tesis Doctoral. Universidad de Almería, 2002.

[15] W. Yu, B. Li, H. Jia, M. Zhang y D. Wang. Application of multi-objective genetic algorithm to optimize energy efficiency and thermal comfort in building design. Energy and Buildings, 88:135-143, 2015. 